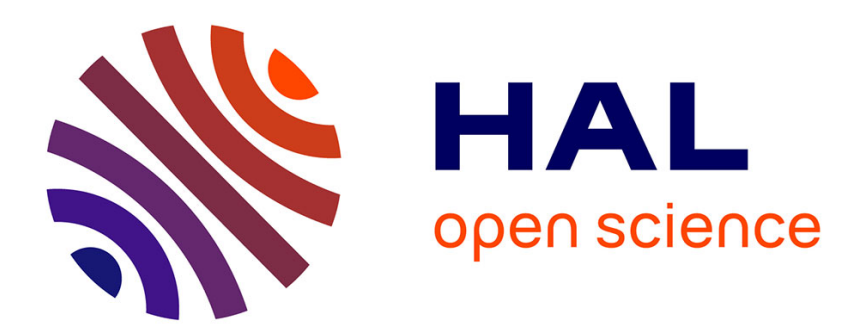

\title{
A VARIATIONAL APPROACH TO THE STUDY OF SURFACE ELECTRONIC PROPERTIES
}

\author{
S. Ying
}

\section{To cite this version:}

S. Ying. A VARIATIONAL APPROACH TO THE STUDY OF SURFACE ELEC-

TRONIC PROPERTIES. Journal de Physique Colloques, 1977, 38 (C4), pp.C4-184-C4-188. 10.1051/jphyscol:1977427 . jpa-00217143

\section{HAL Id: jpa-00217143 \\ https://hal.science/jpa-00217143}

Submitted on 1 Jan 1977

HAL is a multi-disciplinary open access archive for the deposit and dissemination of scientific research documents, whether they are published or not. The documents may come from teaching and research institutions in France or abroad, or from public or private research centers.
L'archive ouverte pluridisciplinaire HAL, est destinée au dépôt et à la diffusion de documents scientifiques de niveau recherche, publiés ou non, émanant des établissements d'enseignement et de recherche français ou étrangers, des laboratoires publics ou privés. 


\title{
A VARIATIONAL APPROACH TO THE STUDY OF SURFACE ELECTRONIC PROPERTIES
}

\author{
S. C. YING $(*)$ \\ Max-Planck-Institut für Festkörperforschung, \\ 7000 Stuttgart 80, Buisnauerstrasse 171, Germany \\ and \\ Brown University (**), Providence, R.I. 02912, U.S.A.
}

\begin{abstract}
Résumé. - Nous proposons une approche variationnelle pour l'étude des propriétés électroniques de surface. La méthode est basée sur une variation directe de la matrice de densité.

On évite les étapes intermédiaires de la théorie self-consistante conventionnelle (calcul des vecteurs propres). La méthode peut être appliquée dans le cas des surfaces propres ainsi que celui des surfaces avec des couches adsorbées.
\end{abstract}

Abstract. - A variational approach to the study of surface electronic properties is proposed. The method is based on a direct variation of the density matrix and bypasses the intermediate steps of searching for eigenvectors in the conventional self-consistent field theory. The method developed is applicable to clean surfaces as well as to surfaces with adsorbed layers.

1. Density functional theory and self-consistent field method. - In the study of electronic properties of inhomogeneous systems such as surfaces with or without adsorbed layers, the density functional theory has played an important role [1]. The basic idea behind this approach is that the total energy $E[n(\mathbf{r})]$ can be expressed as a functional of the density function $n(\mathbf{r})$, the correct value of which minimizes the total energy. Kohn and Sham [2] have shown that the theory can be cast in the form of independent electrons interacting with a selfconsistent field. In recent years there have been many applications of this formalism to the study of surface properties and chemisorption [1]. Most of these are based on the solution of self-consistent field equation using a plane wave basis set, as appropriate for a pseudo-potential description of simple metals and semiconductors. For systems with strong and localized potential such as transition metals, it is more advantageous to cast the density functional theory in a localized orbital representation. We adopt a generalized Wannier function basis consisting of a set of orthonormal orbitals $\phi_{i}(\mathbf{r})$ localized near atomic sites. Here $i$ stands for site index as well as for the type of orbital (e.g. s or d symmetry) on each site. For practical computation, the number of orbitals on each site has

(*) Research supported in part by an Alexander-von-Humboldt Foundation U.S. Senior Scientist award and by a N.S.F. Grant DMR 76-10376.

(**) Permanent Address. to be kept finite. Previous L.C.A.O computations have shown that a limited basis set can provide a realistic description of the ground state properties [3]. The solution to the self-consistent field equation can then be expanded as

$$
\psi_{\alpha \sigma}(\mathbf{r})=\sum_{i} C_{\alpha i \sigma} \phi_{i}(\mathbf{r})
$$

where $\sigma$ is the spin index. The total density is given by

In (2)

$$
n(\mathbf{r})=\sum_{i, j, \sigma} \mathbf{R}_{i j \sigma} \varphi_{i}^{*}(\mathbf{r}) \varphi_{j}(\mathbf{r})
$$

$$
\mathbf{R}_{i j \sigma}=\sum_{\alpha \text { oce. }}^{\prime} C_{\alpha i \sigma}^{*} C_{\alpha j \sigma}
$$

is the density matrix for spin $\sigma$. The summation over $\alpha$ extends through occupied states only. The density matrix defined here is related to the single particle Green's function $G_{i j}(\varepsilon)$ through the equation

$$
\mathbf{R}_{i j \sigma}=-\frac{1}{\pi} \int_{-\infty}^{\varepsilon_{f}} \operatorname{Im} \mathbf{G}_{i j \sigma}(\varepsilon) \mathrm{d} \varepsilon
$$

The matrix element of the effective one electron Hamiltonian in this localized basis set can be formed from the derivative

$$
\mathscr{H}_{i j \sigma}=\frac{\delta E}{\delta \mathbf{R}_{i j \sigma}}=\left\langle\phi_{i}\left|-\frac{\nabla^{2}}{2 m}+V_{\text {ext }}\right| \phi_{j}\right\rangle+\frac{\delta G}{\delta \mathbf{R}_{i j \sigma}}
$$


where we have denoted the part of the total energy that depends on electronic interaction (Coulomb + exchange + correlation) as $G$. In the conventional Hartree-Fock theory,

$G_{\mathrm{HF}}=\sum_{\substack{i, j, k, l \\ \sigma \sigma^{\prime}}}\left\{\begin{aligned} \mathbf{R}_{i j \sigma} & \mathbf{R}_{k l \sigma}{ }^{\prime}\langle i, k|g| l, j\rangle \\ -\mathbf{R}_{i j \sigma} & \mathbf{R}_{k l \sigma} \delta_{\sigma \sigma^{\prime}}\langle i, k|g| j, l\rangle\end{aligned}\right\}$

where

$$
\langle i, k|g| l, j\rangle=\iint \frac{\phi_{i}^{*}(\mathbf{r}) \phi_{k}^{*}\left(\mathbf{r}^{\prime}\right) \phi_{l}\left(\mathbf{r}^{\prime}\right) \phi_{i}(\mathbf{r})}{\left|\mathbf{r}-\mathbf{r}^{\prime}\right|} \mathrm{d} \mathbf{r} \mathrm{d} \mathbf{r}^{\prime}
$$

is the Coulomb matrix element and the second expression in (6) is the exchange term. For application to solids, however, most studies have been based on the local density approximation

$$
G_{l}=\sum_{\substack{i, j, k, l \\ \sigma, \sigma^{\prime}}} \mathbf{R}_{i j \sigma} \mathbf{R}_{k l \sigma^{\prime}}[\langle i, k|g-f| l, j\rangle]
$$

where $f=f(n(\mathbf{r})) \delta_{\sigma \sigma^{\prime}} \delta\left(\mathbf{r}-\mathbf{r}^{\prime}\right)$ depends only on local density. For local exchange alone, $f(n(\mathbf{r})) \propto n^{-1 / 3}(\mathbf{r})$.

Inclusion of local correlation effects requires a more general choice of $f$. In this paper we shall not concern ourselves with the explicit form of $G$ since the formalism to be developed apply for a general functional of the density matrix. We shall also consider paramagnetic systems only and drop the spin indices. Extension to spin dependent properties requires only straightforward generalizations.

The conventional self-consistent field method consists of diagonalizing $\mathbf{H}_{i j}$ in (5) obtained from an approximate initial choice of $\mathbf{R}_{i j}$, generate a new $\mathbf{R}_{i j}$ from the eigenvectors according to (3) and repeating the process until convergence. It is obvious that the procedure is only practical for small molecules or complexes and for systems with high symmetry such as a periodic solid [4]. The direct application of this method to semi-infinite systems cannot be realized even without achieving self-consistency. This motivates the density matrix formalism described below.

2. Direct evaluation of density matrix. - It has long been recognized that the single electron wave functions and eigenvalues obtained from the selfconsistent field equation has no direct physical meaning except as a first approximation for excitation energies and transition probabilities (i.e. neglecting relaxation processes). For the ground state properties it is sufficient to know only the density matrix $\mathbf{R}_{i j}$, the density $n(\mathbf{r})$ then follows from (2) and the energy can be computed from a knowledge of the functional $E\left[\mathbf{R}_{i j}\right]$. Thus in the study of molecules an alternative approach to self-consistent calculations is through the direct variation of the density matrix [5]. The basic problem encountered in the variation of density matrix is that not all its elements are independent [6]. In fact because of its definition in (3) as a projection operator for the vector space of the occupied orbitals, it has the idempotent property

$$
\mathbf{R}^{2}=\mathbf{R} \text {. }
$$

McWeeney [7] has shown that the most general form of variation of $\mathbf{R}$ which preserves idempotency can be written as

$\mathbf{R}+\delta \mathbf{R}=(\mathbf{R}+\lambda \mathbf{V})\left(1+\lambda^{2} \mathbf{V}^{+} \mathbf{V}\right)^{-1}\left(\mathbf{R}+\lambda \mathbf{V}^{+}\right)$,

where

$$
\mathbf{V}=(\mathbf{1}-\mathbf{R}) \Delta \mathbf{R}
$$

In (9), $\Delta$ is an arbitrary matrix of same dimension as $\mathbf{R}$ and $\lambda$ is a multiplicative constant introduced to facilitate separation into different orders. The density matrix can then be determined via unrestricted variation of $\Delta$ to minimize the total energy. Studies on molecular systems have been made based on this approach [8]. Equation (9), as it stands, is not directly useful for an extended system as all the matrices involved have infinite dimensions and matrix multiplication and inversion cannot be achieved. We proceed now to simplify (9) to a form suitable for the study of surface electronic properties. We consider a slab consisting of $N_{0}$ planes of atoms, the limit $N_{0} \rightarrow \infty$ will be made eventually to correspond to two independent surfaces. The system is extended in the direction parallel to the planes and possesses translational symmetry in these dimensions. It is therefore advantageous to Fourier transform all quantities parallel to the surface, for example the density matrix $\mathbf{R}_{i j}$ can be written as

$$
\mathbf{R}_{i j}=\frac{1}{N_{1}} \sum_{\mathbf{k}}\left[\exp i \mathbf{k} \cdot\left(\mathbf{r}_{i}-\mathbf{r}_{j}\right)\right] \mathbf{R}_{u}(\mathbf{k})
$$

where $\mathbf{k}$ and $\mathbf{r}_{i}$ 's are vectors in the plane, and the summation over $\mathbf{k}$ is restricted to the first surface Brillouin zone. The indices $l$ and $l^{\prime}$ stand for different planes as well as for different orbital types on each plane. $N_{1}$ is the total number of atoms on each plane. It is easy to verify that for each $\mathbf{k}, \mathbf{R}_{\mu}$,(k) regarded as a matrix in $l$ and $l^{\prime}$ is idempotent and its most general form of variation is still given by $(8), \mathbf{k}$ playing the role of a parameter only. For the following discussions, we shall suppress the explicit dependence of $\mathbf{k}$ unless necessary. Previous self-consistent calculations [1] have shown that surface charge density, even for a simple metal with extended orbitals, differ from the bulk value only in a highly localized surface region (with the exception of a small amplitude long range Friedel oscillation). The density matrix is therefore expected to have the same property. It is customary to separate the change in the surface properties from the bulk value into a geometric effect and a true surface effect. The former refers to the effects due to the loss of 
neighbours for the surface planes and the latter describes additional charge and bonding relaxation. We write the density matrix as $\mathbf{R}^{\circ}+\delta \mathbf{R}, \mathbf{R}^{0}$ being an idempotent matrix which reduces to the density matrix $\mathbf{R}^{\mathrm{B}}$ of the bulk periodic solid in the interior. It can be chosen so as to include most of the geometric effects. For example, for a non-degenerate s-band, a simple form for $\mathbf{R}^{\circ}[9]$ is

$$
\mathbf{R}_{t^{\prime}}^{0}=\mathbf{R}_{l-l^{\prime}, 0}^{\mathrm{B}}-\mathbf{R}_{i+l^{\prime}, 0}^{\mathrm{B}} \quad\left(l, l^{\prime} \geqslant 1\right) .
$$

There exists a considerable flexibility in the choice of $\mathbf{R}^{0}$. Additional effects can always be included in $\delta \mathbf{R}$. From the above discussion, one expects $\delta \mathbf{R}$ to be nonvanishing mainly in surface region, and hence the matrix $\Delta$ in (9) to be varied should have the same property. The inverse matrix $\left(1+\lambda^{2} V^{+} V\right)^{-1}$ in (9) presents special problems. The variation $\delta \mathbf{R}$, although localized, need not be small, and expansion of the inverse matrix in powers of $\lambda^{2}$ does not necessarily converge and require lengthy evaluations in each order. Moreover, idempontency is not strictly satisfied in a low order expansion and this can lead to serious divergence problems [8]. We propose to accomplish the inversion of $\left(1+\lambda^{2} V^{+} \mathbf{V}\right)$ by restricting the matrix $\Delta$ to the following separable form

$$
\Delta=\lambda \mathbf{F F}^{+}
$$

where $\mathbf{F}$ is a column vector with a finite dimension $p$ corresponding to a finite number of planes in the surface region. Straightforward algebraic manipulations making use of the idempotent property of $\mathbf{R}^{0}$ leads to the following result for $\delta \mathbf{R}$

$$
\begin{aligned}
\delta \mathbf{R}=\frac{1}{1+\lambda^{2} k_{1} k_{2}} & \left\{\lambda \mathbf{F F}^{+(12)}+\lambda \mathbf{F F}^{+(21)}+\right. \\
& \left.+\lambda^{2} k_{1} \mathbf{F F}^{+(22)}-\lambda^{2} k_{2} \mathbf{F F}^{+(11)}\right\}
\end{aligned}
$$

where

$$
\mathbf{F F}^{+(11)}=\mathbf{R}^{0} \mathbf{F F}^{+} \mathbf{R}^{0}, \quad \mathbf{F F}^{+(22)}=\left(1-\mathbf{R}^{0}\right) \mathbf{F F}^{+}\left(1-\mathbf{R}^{0}\right)
$$

and

$$
\begin{aligned}
\mathbf{F F}^{+(12)}=\mathbf{R}^{0} \mathbf{F F} & \left(1-\mathbf{R}^{0}\right), \\
& \mathbf{F F} \\
& =\left(1-\mathbf{R}^{0}\right) \mathbf{F F}^{+} \mathbf{R}^{0}
\end{aligned}
$$

are the components of the matrix $\mathbf{F F}^{+}$that connects vectors within the occupied space and unoccupied space or between the two spaces,

$$
\text { and } \quad k_{1}=\operatorname{Tr} \mathbf{F F}^{+(11)}, \quad k_{2}=\operatorname{Tr} \mathbf{F F}^{+(22)} .
$$

The expression (14) specifies the variation in the density matrix entirely in terms of a vector $\mathbf{F}$ localized in the surface region and it mantains the strict idempotent nature of the varied density matrix $\mathbf{R}^{\circ}+\delta \mathbf{R}$. Thus it is in a suitable form for a surface self-consistent calculation. The form for $\delta \mathbf{R}$ in (9) or (14) indicates that the diagonal and off-diagonal elements of $\mathbf{R}_{i j}$ (often refered to as charge and bond order) are intimately related and cannot be varied independently. It follows from (5) that the same conclusion holds true for elements of the Hamiltonian matrix.

The parameter $\lambda$ measures the magnitude of the variation while the unit vector $F$ determines the optimum direction of variation in the $(\mathrm{k}, l)$ space. To second order in $\lambda^{2}$ the change in energy $\delta E$ due to the variation $\delta \mathbf{R}$ can be expanded as

$$
\begin{gathered}
\delta E=\operatorname{Tr} \mathbf{H} \delta \mathbf{R}+\frac{1}{2} \sum_{i, j, k, l} \frac{\delta^{2} E}{\delta \mathbf{R}_{i j} \delta \mathbf{R}_{k l}} \delta \mathbf{R}_{i j} \delta \mathbf{R}_{k l} \\
\therefore \operatorname{Tr} \overline{\mathbf{H}} \mathbf{F F}^{+}+\frac{\lambda^{2}}{2} A \sum_{l}\left\{\sum_{\mathbf{k}}\left[\mathrm{FF}^{+(12)}+\mathbf{F F}^{+(21)}\right]\right\}_{l l}^{2}
\end{gathered}
$$

In $(17), \overline{\mathbf{H}}$ is the first $p \times p$ block of the matrix $\mathbf{H}^{(12)}(\mathbf{k})+\mathbf{H}^{(21)}(\mathbf{k})$.

In the second order term we have kept only diagonal elements of $\delta \mathbf{R}$ as they are expected to dominate. We have also neglected the small variation of the coefficient $\frac{\delta^{2} E}{\delta R_{i i} \delta R_{i i}}$ over the surface sites and replace it by a constant $A$. This is just the difference of the correlated intraatomic Coulomb and exchange interactions and has been estimated to be positive [10] for typical narrow band solids. Let the eigenvectors of $\overline{\mathbf{H}}$ be denoted by $\mathbf{L}_{1}, \mathbf{L}_{2}, \ldots, \mathbf{L}_{p}$ with eigenvalues $\mu_{1}, \ldots, \mu_{p}$ in decreasing absolute value. We expand $F$ in the form

$$
\mathbf{F}(\mathbf{k})=\sum_{s=1}^{p} x_{s} \mathbf{L}_{s}(\mathbf{k})
$$

The coefficient of $\lambda$ in $\delta E$ is simply equal to $\sum_{\mathbf{k}, s=1}^{p} x_{s}^{2} \mu_{s}(\mathbf{k})$. The second order term is proportional to the quantity

$$
Q^{2}=\sum_{l}\left\{\sum_{k}\left[F^{+(12)}+\mathbf{F F}^{+(21)}\right]\right\}_{u}^{2} .
$$

$Q\left(x_{1}, \ldots, x_{p}\right)$ is a measure of the surface charge deviation from the original state. The optimum $F$ corresponds to the set $\left\{x_{1}, \ldots, x_{p}\right\}$ which maximizes the ratio $r=\left|\sum_{k, s} x_{i}^{2} \mu_{s}(\mathbf{k})\right| / Q$. This scheme allows the variation $\delta \mathbf{R}$ to avoid excited states involving large charge fluctuations and is clearly superior to the steepest descent path $\mathbf{F}=\mathbf{L}_{1}$. The value of $\lambda$ which minimizes $\delta E$ follows from (17) as

$$
\lambda_{\mathrm{o}}=-r^{2}\left(A \sum_{\mathbf{k} s} x_{s}^{2} \mu_{s}(\mathbf{k})\right)^{-1}
$$

The density matrix $\mathbf{R}^{0}+\delta \mathbf{R}\left(\lambda_{0}\right)$ determined this way is expected to give a good description of the ground state when $\delta \mathbf{R}$ is small. If the initial density matrix $\mathbf{R}^{o}$ corresponds to a highly excited state this would not be the case. Then one can replace $\mathbf{R}^{\circ}$ by the new matrix $\mathbf{R}^{0}+\delta \mathbf{R}\left(\lambda_{0}\right)$ and repeat the procedure until the energy converges. In this iteration scheme, there is no loss of generality in choosing a separable form 
for the variational matrix $\Delta=\mathbf{F F}^{+}$as well as restricting $\mathbf{F}$ to have only a finite number of surface components. We note that in the present formulation the surface region has been reduced to an effective thin film of $p$ layers with $\overline{\mathbf{H}}$ playing the role of an effective surface Hamiltonian. However, the actual change in the density matrix $\delta \mathbf{R}$ is not limited to the first $p$ planes as can be seen from equation (14) and (15). The surface information is propagated into the interior through the projection operators $\mathbf{R}^{\circ}$ and $1-\mathbf{R}^{\circ}$. The necessary input from the bulk calculations is contained in the asymptotic form for $\mathbf{R}^{0}$.

3. Surfaces with adsorbed layers. - Since the surface region has been reduced to an effective thin film, an extra layer of adatoms can be easily studied with the same formalism provided the system still possesses translational symmetry in the dimensions parallel to the surface. For simplicity we shall consider here only the case of an epitaxial layer of adatom with a doubly occupied orbital interacting with the substrate. First additional orbitals have to be introduced, a simple choice would be the atomic orbital for the adatom orthogonalized to each other in the surface plane and to the substrate orbitals. More general Wannier functions can be constructed via variational techniques [11]. Denote the density matrix for the clean substrate as $\mathbf{R}_{s}$ and consider the following density matrix

$$
\mathbf{R}^{\circ}(\mathbf{k})=\left(\begin{array}{cc}
1 & 0 \\
0 & \mathbf{R}^{s}(\mathbf{k})
\end{array}\right)
$$

$\mathbf{R}^{\circ}(\mathbf{k})$ is obviously idempotent following the idempotency of $\mathbf{R}^{s}$. Physically, $\mathbf{R}^{\mathbf{0}}$ describes an isolated adsorbed layer and a substrate with no bonding between them. Their self-consistent bonding can then be described again through the introduction of a variation in the density matrix $\delta \mathbf{R}$ of the form prescribed by (14). As each additional layer is adsorbed, we expect the readjustment of the charge and bond order to be localized in the neighbourhood of the extra plane. An initial choice of the density matrix such as that given by (21) makes good use of the existing surface informations just before the last layer is adsorbed.

Thus far we have assumed implicitly that the localized basis set for the clean substrate on each plane is the same as that of the bulk solid. The optimum surface orbital should involve additional distortions as expected from a renormalized atom argument [12]. An alternate way of expressing this fact is that hybridization with additional orbitals becomes important in the surface region. This hybridization can be achieved by introducing an extra plane of localized orbitals at a variable distance $d$ above the last atomic plane in the same manner as in the study of an additional adsorbed layer. The nature of these orbitals would depend on the surface potential. A useful input potential is that determined from a surface calculation without orbital distortion. The initial density matrix in this case can be chosen as

$$
\mathbf{R}^{\mathbf{0}}=\left(\begin{array}{cc}
0 & 0 \\
0 & \mathbf{R}^{s}
\end{array}\right)
$$

and the hybridization with the virtual orbitals again described by self-consistent variation of $\delta \mathbf{R}$.

In the limit $d \rightarrow 0$, the above procedure corresponds to introducing additional atomic orbitals on the surface site into the basis set. The advantage of locating these orbitals at an extra plane is that the additional orbitals can be of higher symmetries. For example, a p-like distortion of the surface orbital can be described by an additional s orbital in the extra layer. It also allows a unified treatment of substrates, adsorbed layers and surface hybridization effects.

4. Summary. - We have described a variational approach to the study of surface electronic properties. It is based on a direct study of the density matrix rather than the eigenvectors of an extended system in the presence of a surface. Since the former contains less informations, it should in principle be easier to determine. The surface problem is divided into successive steps. In the study of the clean substrate, the bulk properties are taken as known input. It determines the asymptotic form of the density matrix. The density matrix for the clean substrate is then used as an input for subsequent adsorption processes. This step by step procedure allows one to reduce the surface region into an effective thin film at any given stage. It is ideally suited for the study of transition from monolayer adsorption to bulk properties of the adsorbate as successive layers are adsorbed.

Acknowledgment. - The author acknowledges helpful discussions with P. Fulde and K. P. Bohnen. He would also like to thank the hospitality of the Max-Planck-Institut für Festkörperforschung at Stuttgart and the support of Alexander von Humboldt Foundation during the period where the work was performed.

\section{References}

[1] LANG, N., Solid State Phys. (éd. Seitz \& Turnbull) 28 (1974), 225.

[2] Kohn, W. \& Sham, L., Phys. Rev. 140 (1965) 1133.
[3] Chaney, R. C., Tung, T. K., Lin, C. C., Lafon, E. E., $J$. Chem. Phys. 52 (1970) 361.

[4] Gay, J. G., Smith, J., Arlinghaus, F. J., Phys. Rev. Lett. 
38 (1977) 561. Recently reported a self-consistent calculation on thin films of $\mathrm{Cu}$.

[5] a) McWeeney, R., Proc. R. Soc. A 235 (1956) 496.

b) Hiller, I. H. and SAUnders, V. R., Int. J. Quantum Chem., IV (1970), 503.

[6] Smith, J. and GaY, J. G., Phys. Rev. 12 (1976), 4238

[7] McWeEneY, R., Rev. Mod. Phys. 32 (1960), 35.

[8] Sleeman, D. H., Theor. Chim. Acta. 11 (1968), 135.
[9] Allan, G., Ann. Phys. (Paris) 5 (1970), 166.

[10] FRIEDEL, J., Physics of Metals (Ed. Ziman J.) 1969, 340.

[11] a) KoHN, W., Phys. Rev. B 1 (1973) 4388.

b) Smith, J. R. and GAY, J. G., Phys. Rev. Lett. 32 (1974) 774 .

[12] Fulde, P., Luther, A., Watson, R. E., Phys, Rev. B 8 (1973), 440.

\section{DISCUSSION}

A. D. NOVACO. - What does one take for the exchange correlation functional ?

S. C. YING. - The usual approximation is the local exchange correlation functional which is derived from the theory of the uniform electron gas. The X- $\alpha$ approximation is an additional approximation on the correlation energy.
E. BAUER. - The convergence of cluster on thin film calculation as a function of the size of the system depends very much on the individual character of the system. In the case of $\mathrm{Na}$ slab that we have studied, the kinetic energy is not yet convergent even for a film of 14 layer thickness. 